\title{
An unusual case of small intestinal tuberculosis with enteroenteric fistula: A case report
}

\author{
Uma Debi ${ }^{1}$, Abhinaya Shankar ${ }^{1}$, MV Kartik ${ }^{1}$, Kaushal Kishor Prasad ${ }^{2}$, BR Thapa ${ }^{2}$ \\ ${ }^{1}$ Department of Radiodiagnosis, ${ }^{2}$ Department of Gastroenterology \\ Postgraduate Institute of Medical Education and Research, Chandigarh, India
}

\begin{abstract}
Tuberculosis is a very common condition which at times can present with distinctly atypical findings. In the vast majority of the presenting cases it can pose diagnostic challenge to both the clinician and the radiologist due to its nonspecific clinical manifestations and lack of pathognomonic radiological signs But being a treatable disease utmost care needs to be exercised in establishing this diagnosis. We report a rare radiological finding in a case of small intestinal tuberculosis which confounded the initial diagnosis necessitating pathological proof of tubercular enteroenteric fistula. Following fine needle aspiration cytology, the patient was started on anti-tubercular treatment and conservatively managed. The patient showed significant improvement in clinical symptoms after 3 months and resolution on completion of the treatment.
\end{abstract}

Keywords: Enteroenteric fistula, FNAC, intestinal tuberculosis, ultrasonography

\section{INTRODUCTION}

Tuberculosis is a common health problem with a rising trend in prevalence fuelled by several important factors in the current global health scenario, the most important being the AIDS pandemic. Both pulmonary and extrapulmonary cases of tuberculosis are well known with the latter usually non specific in clinical and sometimes even in its radiological presentation, necessitating tissue sampling to establish a diagnosis. ${ }^{[1]}$ Only approximately $15-25 \%$ of cases with abdominal tuberculosis have concomitant pulmonary tuberculosis ${ }^{[2,3]}$ which can accelerate the diagnosis. The main entities that need distinction from this infectious pathology are lymphoma and inflammatory bowel disease (particularly Crohn's disease). We report a rare case of small intestinal tuberculosis which had features of both these conditions.

Corresponding Author: Dr. Uma Debi

E-mail: debi_uma@yahoo.co.in

Received: 09-08-2015

Accepted:16-11-2015

How to cite this article: Debi U, Shankar A, Kartik MV, Prasad KK, Thapa BR. An unusual case of small intestinal tuberculosis with enteroenteric fistula: A case report. J Gastrointest Infect, 2015; 5: 46-49.

\section{CASE REPORT}

A 14 year old immunocompetent (non-HIV) female patient presented with generalized abdominal pain which at times was colicky in nature, associated with nausea, vomiting and constipation for a duration of 6 months. She had loss of appetite, loss of weight, and low grade fever since three months. There was no history of anti-tubercular treatment (ATT) intake or contact with any case of tuberculosis. There was no other significant co-morbidity. Abdominal examination revealed distention without any localized tenderness or palpable lump. Other system examination was unremarkable. Routine blood examinations revealed anemia ( $\mathrm{Hb} 9.6 \mathrm{~g} \%)$, elevated erythrocyte sedimentation rate (ESR). Chest radiograph was normal. Ultrasonography of the abdomen revealed mesenteric lymphadenopathy and mild ascites (Fig. 1a \& 1b). Ascitic serum adenosine deaminase (ADA) level was $44 \mathrm{U} / \mathrm{L}$.

On contrast enhanced computed tomography (CECT) of the abdomen, additional findings included high density ascites (mean HU 30) peritoneal and omental thickening and diffuse small bowel (predominantly ileal) thickening with intramural 

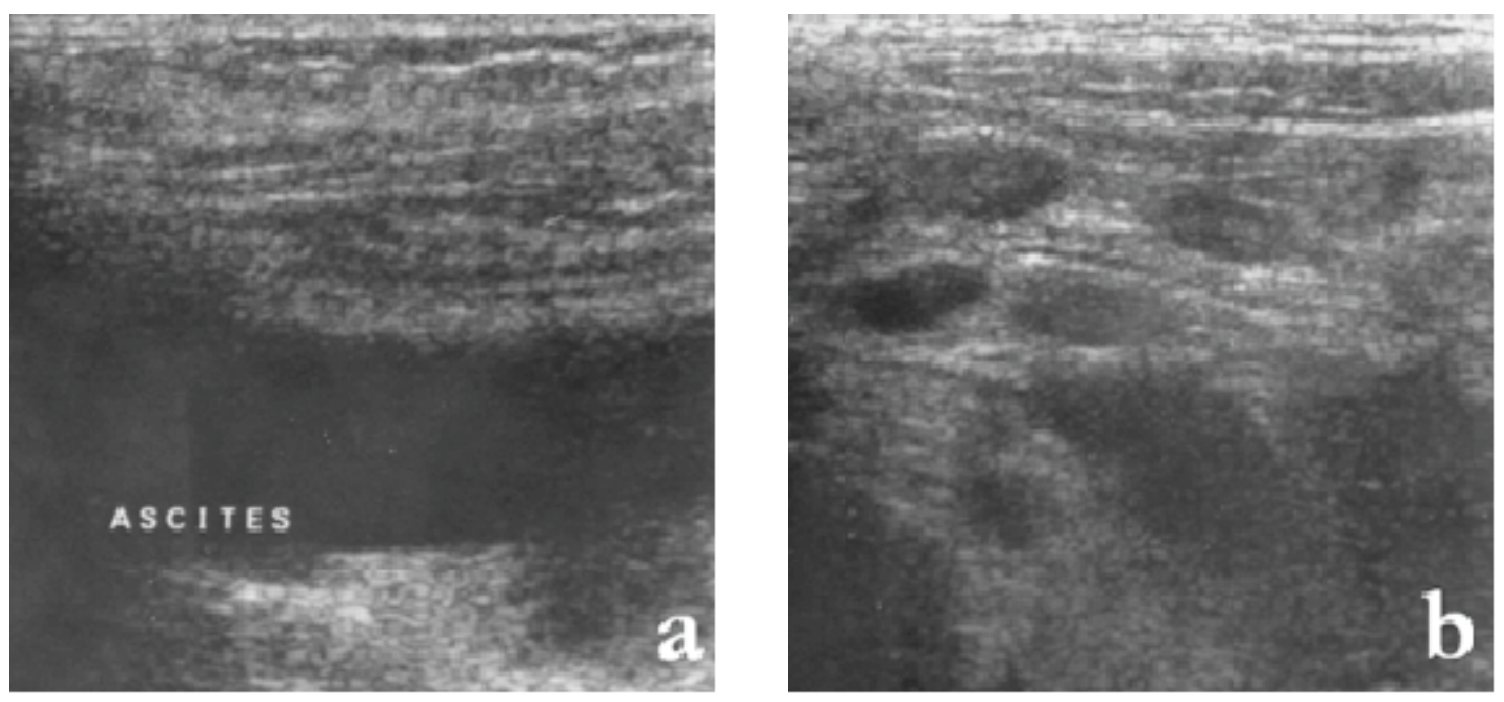

Fig. 1a \& 1b: Ultrasound images showing mesenteric lymphadenopathy (few of them showing necrosis) and ascites.

necrotic foci in right iliac fossa. In addition there was an interbowel communication through a soft tissue lined fistulous tract traversing the mesentery (Fig. $2 a \& 2 b$ ). The ileocecal junction was normal, with mild cecal wall thickening. Barium meal follow through (BMFT) was performed which confirmed fistulization and depicted multiple ileal strictures (Fig. 3a \& 3b). As diffuse wall thickening was visualized on ultrasound so fine needle aspiration cytology (FNAC) smear from the bowel wall thickening and mesenteric soft tissue was performed which revealed granulomatous inflammation and was positive for acid fast bacilli stain. A CT guided omental biopsy was not performed.

A final diagnosis of intestinal tuberculosis with enteroenteric fistula was made and ATT was started. Resolution of patient symptoms were seen after completion of ATT and repeat BMFT showed no interbowel communication. The patient showed significant improvement in clinical symptoms after 3 months and resolution on completion of the treatment. The patient recovered and was doing well on follow-up after 2 years.

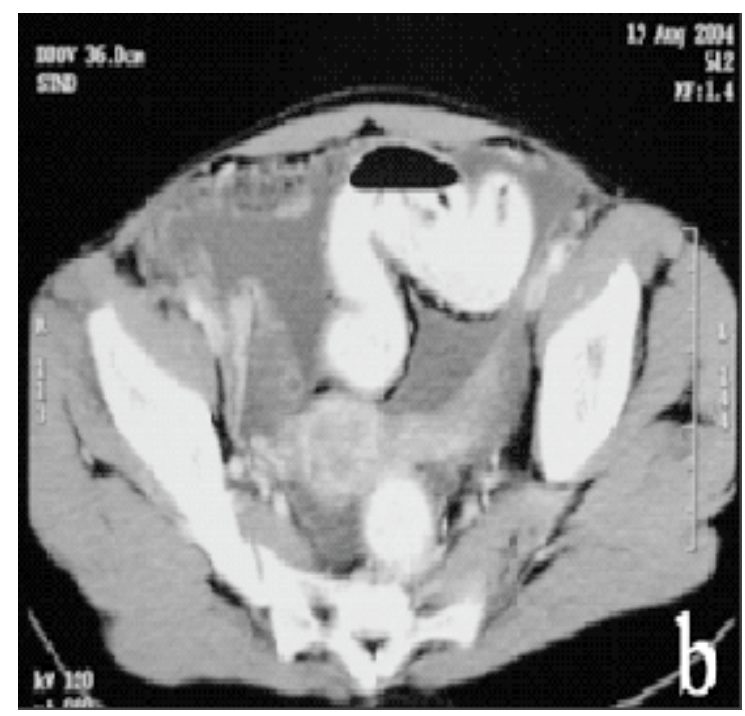

Fig. 2a \& 2b: CECT images showing high density ascites, small bowel wall thickening with fistulous tract lined with soft tissue, smooth peritoneal thickening and enhancement. 

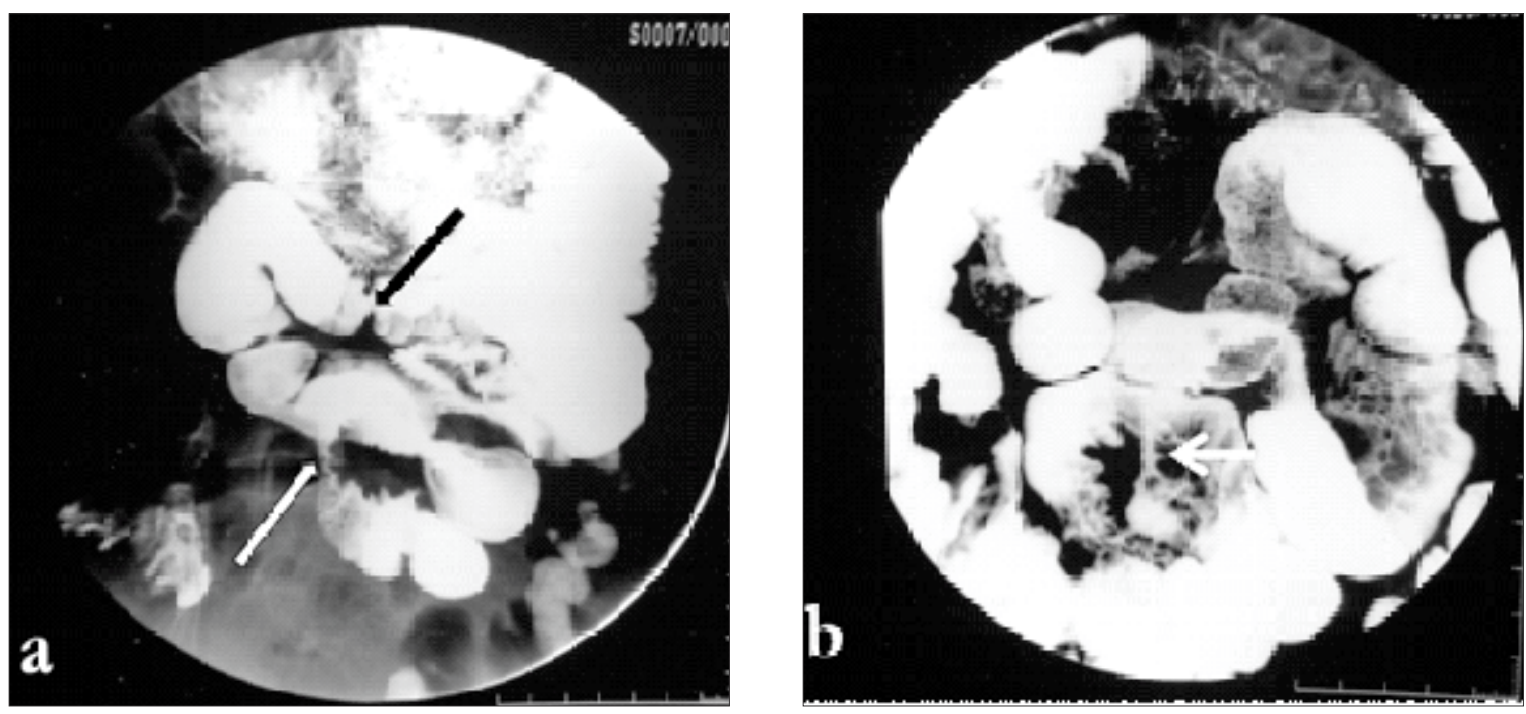

Fig. 3a \& 3b: BMFT images showing lleal strictures and inter bowel fistulous.

\section{DISCUSSION}

Abdominal tuberculosis constitutes 11\% of the extrapulmonary cases. ${ }^{[4]}$ Four major types of abdominal tuberculosis are described: nodal, visceral, gastrointestinal and peritoneal. The most common site of gastrointestinal tuberculosis is terminal ileumcecum complex, followed by jejunum and colon. ${ }^{[5]}$ The ileocecal region is involved in $64 \%$ of cases of gastrointestinal tuberculosis. ${ }^{[6]}$ This is because of the various contributing factors like stasis, presence of abundant lymphoid tissue in the terminal ileum, increased rate of absorption at this site and closer contact of the bacilli with the mucosa. ${ }^{[2,7,8]}$ Even though fistulous communication is more common in Crohn's disease, in gastrointestinal tuberculosis, on rare occasion enteroenteric fistula can occur.

Irrespective of the site of affliction the pathological process remains the same with three major subtypes: ulcerative, hypertrophic and a third type having features of both. The disease goes through active inflammatory and burnt out fibrotic phases. During the healing/fibrotic stage of the disease multiple complications may occur due to loss of pliability of the bowel wall; the most common complication being bowel obstruction secondary to stricture formation or due to adhesions. ${ }^{[9]}$ Other rarer complications of intestinal tuberculosis include intestinal perforation
$(4.9 \%)$, enterocutaneous fistula formation $(2.4 \%)$ and small bowel volvulus due to mesenteric lymphadenitis $(2.4 \%){ }^{[10]}$

Onkar et $a l^{[10]}$ reported a case of spontaneous tubercular enterocutaneous fistula developing a surgical scar 14 years after an appendicectomy. But to the best of our knowledge there has been no reported case of tubercular enteroenteric fistula in a case of primary extrapulmonary tuberculosis. In our index case, there were systemic symptoms but no extraabdominal focus could be identified. Although there were findings typical of tuberculosis including high density ascites, peritoneal and omental thickening with mesenteric lymphadenopathy, there were a few unusual findings including sparing of the ileocecal region with more proximal small bowel (ileal) involvement, a well defined fistulous tract between two ileal loops. Fistulas are more commonly encountered in small bowel lymphoma and Crohn's disease. To the best of our knowledge, enteroenteric fistula in extrapulmonary gasrtrointestinal tuberculosis is not reported in English literature. This highlights the fact that indeed tuberculosis is a great mimic of other inflammatory and neoplastic diseases of the small bowel. In areas where this disease is endemic, it should always be included in the differential diagnosis of bowel wall thickening with peritoneal involvement even with atypical radiological findings. 


\section{REFERENCES}

1. Mukewar S, Mukewar S, Ravi R, Prasad A, S Dua K. Colon tuberculosis: endoscopic features and prospective endoscopic follow-up after anti-tuberculosis treatment. Clin Transl Gastroenterol. 2012; 3:e24.

2. Horvath $\mathrm{KD}$, Whelan RL. Intestinal tuberculosis: return of an old disease. Am J Gastroenterol. 1998; 93:692-6.

3. Akhan $\mathrm{O}$, Pringot J. Imaging of abdominal tuberculosis. Eur Radiol 2002;12:312-23.

4. Kapoor V K. Abdominal tuberculosis. Postgrad Med J. 1998;74:459.

5. Debi U, Vasudevan R, Prasad KK, Sinha SK, Sharma AK. Abdominal tuberculosis of the gastrointestinal tract: Revisited. World J Gastroenterol. 2014; 20:4831-40.

6. Sharma R. Abdominal Tuberculosis. Imaging Science Today
2009: 146. Available from: URL: http://www. imagingscience today.com/node/146.

7. Alvares JF, Devarbhavi H, Makhija P, Rao S, Kottoor R. Clinical, colonoscopic, and histological profile of colonic tuberculosis in a tertiary hospital. Endoscopy. 2005;37:351-6.

8. Das HS, Rathi P, Sawant P, Chodankar CM, Vyas K, Patrawala V Dhadphale S. Colonic tuberculosis: colonoscopic appearance and clinico-pathologic analysis. J Assoc Physicians India. 2000;48:708-10.

9. McGuinness FE, Hamilton D, Al Nabulsi J. Tuberculosis of the gastrointestinal tract and peritoneum. In: McGuinness FE, editor. Clinical imaging of non-pulmonary tuberculosis. Berlin: Springer. 2000:107-38.

10. Singh O, Gupta S, Moses S, Jain DK. Spontaneous tubercular enterocutaneous fistula developing in the scar of a surgery done 14 years earlier. Saudi J Gastroenterol. 2009;15:261-38. 